\section{Ohio State's pep(tide) rally}

\section{By Kai-Jye Lou, Staff Writer}

A group at The Ohio State University Medical Center has advanced a pipeline of peptide-based cancer vaccines and therapeutics into preclinical studies and Phase I trials with $\$ 9.6$ million in funding from NIH, NCI, the university and not-for-profit organizations such as the Susan G. Komen Breast Cancer Foundation. The most advanced program-a HER2 vaccine-has completed Phase I testing, and the researchers plan to start a Phase I trial to evaluate their second-generation vaccine next week.

The group is now seeking industry partners to accelerate Phase II development and to help move its remaining preclinical programs into the clinic.

The team, led by Pravin Kaumaya, a professor in the Department of Obstetrics and Gynecology at OSU and director of the Division of Peptide Therapeutics and Cancer Vaccine Research, has been building a pipeline of engineered peptide mimics against validated cancer targets like epidermal growth factor receptor 1 (EGFR1; HER1; ERBB1), HER2 (EGFR2; ERBB2; neu) and VEGF.

The OSU group thinks its peptide mimics could have a safety and specificity advantage over small molecules as well as lower cost and less potential for resistance than $\mathrm{mAbs}$.

The group started in 1995 by mapping out the key immunogenic epitopes of the HER2 oncoprotein and synthesizing and evaluating peptide B cell epitopes for its first-generation HER2 vaccine candidate. Today, the division's pipeline includes eight programs evaluating B cell epitopes and peptide mimics as cancer vaccines and therapeutics.

The peptide $B$ cell epitopes used in the vaccine candidates are synthesized together with a $\mathrm{T}$ cell epitope from the measles virus fusion protein (MVF protein), which causes the body to mount an immune response against the attached $\mathrm{B}$ cell peptide. The therapeutic peptide mimics lack the immunogenic $\mathrm{T}$ cell epitope and instead work by directly disrupting a cancer target's function.

The group's first-generation HER2 vaccine contains two HER2 epitopes conjugated to an immunogenic epitope of the MVF protein. In a Phase I trial in 24 stage IV cancer patients in 2007, the vaccine produced no serious adverse events, autoimmune reactions or cardiotoxicity. ${ }^{1}$ There were two partial responses and four cases of stable disease.

OSU's second-generation vaccine was designed based on the known $3 \mathrm{D}$ structure of the oncoprotein and uses a different but overlapping pair of HER2 epitopes conjugated to the MVF epitope.

Kaumaya said the group's vaccines are active immunotherapies that cause the body's B cells to generate an endogenous polyclonal antibody response that disrupts HER2 signaling. In contrast, drugs like Herceptin trastuzumab work by disrupting HER2 signaling directly.

He thinks the active immunotherapy approach for keeping cancer in remission is a better option than weekly $\mathrm{mAb}$ infusions because the vaccine prompts the patient's immune system to constantly generate antibodies against the target.

In the four-year Phase I study, patients received a total of three subcutaneous inoculations with the HER2 vaccine with an adjuvant. In addition, Kaumaya thinks the vaccine could be used in the prophylactic setting in genetically predisposed individuals.

Herceptin is given weekly or once every three weeks via i.v. infusion.

Maurizio Zanetti, a professor of medicine at the University of

Table 1. Ohio State University's peptide-based cancer programs. The Division of Peptide Therapeutics and Cancer Vaccine Research in the Department of Obstetrics and Gynecology at The Ohio State University Medical Center is taking a multi-pronged approach to cancer treatment that includes vaccines consisting of peptide B cell epitopes conjugated to a T cell epitope that increases the immune response, stand-alone peptide mimic therapeutics and combinations of the two.

Source: Pravin Kaumaya, The Ohio State University Medical Center

\begin{tabular}{ll}
\hline Program description & Status \\
\hline $\begin{array}{l}\text { Virst-generation product containing two peptide B cell epitopes of HER2 (EGFR2; ERBB2; neu), } \\
\text { each conjugated to a T cell epitope of the measles virus fusion protein (MVF protein) }\end{array}$ & \\
\hline $\begin{array}{l}\text { Second-generation product containing two peptide B cell epitopes of HER2, each conjugated } \\
\text { with a T cell epitope of the MVF protein }\end{array}$ & Phase I \\
\hline B cell epitope of VEGF conjugated to an undisclosed T cell epitope & Preclinical testing \\
\hline Therapeutic peptides & \\
\hline Mimics of a VEGF epitope & Preclinical testing \\
\hline Mimics of a HER1 (EGFR1; ERBB1) epitope & Preclinical testing \\
\hline Mimics of a HER3 (EGFR3; ERBB3) epitope & Preclinical testing \\
\hline Combinations & \\
\hline HER2 vaccine plus a VEGF peptide mimic & Preclinical testing \\
\hline HER2 vaccine plus a VEGF peptide mimic and paclitaxel & Preclinical testing \\
\hline HER2 peptide mimic and a VEGF peptide mimic & Preclinical testing
\end{tabular}


California, San Diego, liked OSU's active immunotherapy approach.

"It is my opinion that the polyclonal antibody response produced following vaccination would produce a better response than a single-targeting $\mathrm{mAb}$ " because it could be more difficult for the cancer to develop escape or resistance mechanisms against an entire set of antibodies, he told SciBX.

Zanetti also said OSU's HER2 vaccines "could lead to improvements in the patient's quality of life by reducing the frequency of treatment and also have reduced cost" compared with Herceptin.

Herceptin, from Roche's Genentech Inc. unit, is marketed to treat HER2-overexpressing breast cancers and metastatic gastric cancers. The drug costs about $\$ 4,200$ per month in the U.S. Kaumaya expects OSU's HER2 vaccines will be cheaper and easier to manufacture than antibodies, and he suggested a single treatment could cost as little as $\$ 300-\$ 400$.

\section{The therapeutic option}

Although the OSU group started with peptide-based cancer vaccines, it has expanded its scope to include therapeutic peptide mimics for cancer.

"Previously, peptide-based therapeutics were rapidly proteolyzed and degraded, which resulted in poor efficacy," said Kaumaya. "In our current pair of studies, we engineered peptide mimics that are resistant to proteolysis in vivo by synthesizing the epitope using $\mathrm{D}$-amino acids in reverse order. This synthesis method has been shown to increase the half-life of the peptides, which can lead to improvements in efficacy."

Last month, the OSU group published a pair of papers in The Journal of Biological Chemistry describing the synthesis and evaluation of VEGF peptides that mimic the binding of VEGF to VEGF receptor 2 (KDR/Flk-1; VEGFR-2) as a stand-alone treatment ${ }^{2}$ and in combination with a previously reported HER2 mimic for cancer. ${ }^{3}$

Several of the therapeutic peptides in the $J B C$ papers were synthesized in the direction opposite of normal peptide synthesis.

Kaumaya said the group's peptides should have minimal immunogenicity issues because they are based on epitopes of native human protein. He added that the therapeutic peptides are designed to have a safety and specificity advantage over small molecules and should not cost more than $\$ 1,000$ per treatment.

"Small molecule drugs like Sutent are known to have many side effects and improve survival by only a few months," he told SciBX. "These side effects are believed to be due to their promiscuous inhibitory activity. Peptide-based therapeutics are much more specific for their targets than small molecules."

Pfizer Inc.'s Sutent sunitinib, a small molecule VEGF receptor tyrosine kinase inhibitor, is marketed to treat gastrointestinal stromal tumors (GIST) and advanced renal cell carcinoma (RCC).

"Most people agree that Sutent's side-effect profile is due to its spectrum of kinase inhibition on targets other than VEGF receptors 1, 2 and 3," noted Murray Robinson, SVP of translational medicine at Aveo Pharmaceuticals Inc.

Aveo and partner Astellas Pharma Inc. are developing Tivozanib (AV-951), a small molecule against VEGF receptor 1 (FLT1), VEGFR-2 and VEGF receptor 3 (FLT4). The compound is in Phase III testing for RCC with data expected mid-year. The biotech licensed ex-Asia rights to Tivozanib from Kirin Brewery Co. Ltd. in 2007.

Robinson thinks Tivozanib's selectivity and potency will help it overcome the issues associated with previous small molecule drugs like Sutent.

"Tivozanib exhibits 160-240 picomolar inhibitory activity against VEGF receptors 1, 2 and 3, with 8 - to 10 -fold greater potency against the VEGF receptors than the next most potent measured kinase," he told SciBX. "We believe that this potency and selectivity directly translates to the observed efficacy and toxicity profile that we have reported in our Phase II renal cell carcinoma study, where the progression-free survival observed was the longest reported in clear cell RCC."

\section{Partner up}

Thus far, Kaumaya's group has received a total of $\$ 9.6$ million and advanced its programs into Phase I trials and late-stage preclinical development. Kaumaya said the goal is to find industry partners to advance the lead HER2 vaccine and combination mimics program into Phase II testing. He estimates that a limited, 45-60 patient Phase IIa trial in a single indication will cost between \$2-\$3 million if it is carried out at the OSU Comprehensive Cancer Center.

He added that the group will need $\$ 2-\$ 3$ million to move its preclinical HER2 vaccine or HER2 mimic plus VEGF mimic combination programs into Phase I testing. The group's program evaluating a triple combination of its HER2 vaccine and VEGF mimic plus paclitaxel will require another $\$ 2$ million to advance into Phase I, he said.

The group also has a VEGF peptide vaccine program as well as HER1 and HER3 (EGFR3; ERBB3) therapeutic peptide mimics in preclinical development for cancer, but it has not disclosed further details.

OSU has multiple issued patents covering its peptide mimic vaccines and therapeutics (see Table 1, “Ohio State University's peptide-based cancer programs"). The work is available for licensing.

Lou, K.-J. SciBX 4(10); doi:10.1038/scibx.2011.272

Published online March 10, 2011

\section{REFERENCES}

1. Kaumaya, P.T.P. et al. J. Clin. Oncol. 27, 5270-5277 (2009)

2. Vicari, D. et al. J. Biol. Chem.; published online Feb. 14, 2011; doi:10.1074/jbc.M110.216812

Contact: Pravin T.P. Kaumaya, The Ohio State University Medical Center, Columbus, Ohio e-mail: kaumaya.1@osu.edu

3. Foy, K.C. et al. J. Biol. Chem.; published online Feb. 16, 2011; doi:10.1074/jbc.M110.216820

Contact: Pravin T.P. Kaumaya, The Ohio State University Medical Center, Columbus, Ohio

e-mail: kaumaya.1@osu.edu

\section{COMPANIES AND INSTITUTIONS MENTIONED}

Astellas Pharma Inc. (Tokyo:4503), Tokyo, Japan

Aveo Pharmaceuticals Inc. (NASDAQ:AVEO), Cambridge, Mass. Genentech Inc., South San Francisco, Calif.

Kirin Brewery Co. Ltd. (Tokyo:2503; Pink:KNBWY), Tokyo, Japan

The Ohio State University Medical Center, Columbus, Ohio

Pfizer Inc. (NYSE:PFE), New York, N.Y.

Roche (SIX:ROG; OTCQX:RHHBY), Basel, Switzerland

Susan G. Komen Breast Cancer Foundation, Dallas, Texas University of California, San Diego, La Jolla, Calif. 